\title{
Capsule Commentary on Tomko et al., A Comparison of Web- Versus Print-Based Decision Aids for Prostate Cancer Screening: Participants' Evaluation and Utilization
}

\author{
Elizabeth Kessler, MD \\ DOI: 10.1007/s11606-014-3039-y \\ (c) Society of General Internal Medicine 2014
}

Division of Medical Oncology, University of Colorado Anschutz Medical Campus, University of Colorado Cancer Center, Aurora, CO, USA.
C. Tomko et al. ${ }^{1}$ compares web-based and print-based decision tools for prostate cancer screening. Using an already supported prostate cancer screening decision aid, they asked the practical question of how these tools are best implemented. In their cohort of 1,235 adults, decision aid use was independently influenced by race, education, and the decision aid medium. The best choice based on preference and utilization was print medium. Thus, in an increasingly digitized age, there is still a role for paper and pencil.

Decision aids are helpful when patients are faced with complex decisions with no clear-cut "best answer" and uncertain ramifications. There has been much discussion regarding the utility of prostate cancer screening with digital rectal exam and a serum prostate specific antigen (PSA), with most organizations recommending against routine screening. ${ }^{2}$ However, patients are still being screened under the rubric that this should be a shared decision thaht occurs after discussion between patient and provider. However, this decision has been shown to be biased by multiple factors on the part of the provider and patients, ${ }^{3}$ and the accuracy and amount of information provided is variable. ${ }^{4}$ Decision tools may be helpful and effective in these situations, by providing accurate evidence-based information to support decision-making.

The current study is interesting not so much in that it adds to the support of using decision aids, but that it addresses how best to implement these effective tools. If we are to work to impact patient experience, we need to understand how best to apply evidence-supported method- ology to real-world scenarios. ${ }^{5}$ Our medical systems have turned increasingly to electronic health records, and informational web portals; yet, this study concludes that print may be a preferred medium for many patients. An important factor for some patients is still internet access and computer skills. The "digital divide" still exists and requires recognition as these tools are implemented. The clearest implication from this study is that in an age of patient choice, even the format of the decision aid should be up to the patient.

Conflict of Interest: The author declares that she does not have a conflict of interest.

Corresponding Author: Elizabeth Kessler, MD; Division of Medical Oncology, University of Colorado Anschutz Medical Campus, University of Colorado Cancer Center, MS 8117, 12801 E 17th Ave, Aurora, CO 80045, USA (e-mail: Elizabeth.kessler@ucdenver.edu).

\section{REFERENCES}

1. Tomko C, Davis KM, Luta G, Krist AH, Woolf SH, Taylor KL. Comparison of Web- versus Print-Based Decision Aids for Prostate Cancer Screening: Participants' Evaluation and Utilization J Gen Intern Med. doi: 10.1007/ s11606-014-2994-7.

2. Moyer VA. Screening for Prostate Cancer: U.S. Preventive Services Task Force Recommendation Statement. Ann Intern Med. 2012;157(2):I-44.

3. Woods VD, et al. Social ecological predictors of prostate-specific antigen blood test and digital rectal examination in black American men. J Natl Med Assoc. 2006;98(4):492-504.

4. Konety BR, et al. Effect of uniform consensus recommendations for PCa screening in older population: differential effects and perceptions of healthcare providers and patients. Urology. 2009;73(3):603-608. discussion 608-93.

5. Oakley A, et al. Process evaluation in randomised controlled trials of complex interventions. BMJ. 2006;332(7538):413-416.

Published online September 30, 2014 